\title{
PROACTIVIDAD Y CONTEXTUALIZACIÓN: FUTURO DEL DISEÑO DE SISTEMAS RECOMENDADORES
}

\author{
Daniel Gallego-Vico, Antonio Fumero-Reverón y Gabriel Huecas-Fernández- \\ Toribio
}

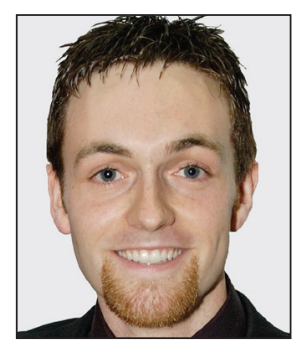

Daniel Gallego-Vico es ingeniero de telecomunicación y máster en ingeniería de redes y servicios telemáticos, ambos por la Universidad Politécnica de Madrid. Trabaja como investigador doctoral en la misma universidad dentro del Grupo de Internet de Nueva Generación. Investiga la aplicación de sistemas de recomendación proactivos basados en información contextual derivada del análisis de datos sociales, físicos y de usuario, además de analizar el impacto de dichos sistemas en la experiencia de usuario. Es socio fundador de Zombies in the lab y participa en iniciativas empresariales dentro del mundo emprendedor.

http://orcid.org/0000-0002-4163-9850

dgallego@dit.upm.es

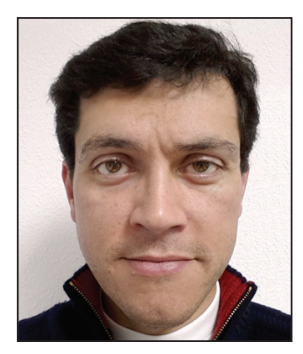

Antonio Fumero-Reverón es ingeniero de telecomunicación y MBA por la Universidad Politécnica de Madrid (UPM). Especialista en gestión de la tecnología e innovación, comenzó su carrera profesional en la Unidad de Desarrollo Tecnológico e Innovación del BBVA. Es investigador en la UPM, colaborando con la Cátedra Telefónica para Internet de Nueva Generación y el Grupo de Tecnologías de la Información para la Gestión Empresarial. Es socio fundador de Win-Win Consultores, y participa en otras iniciativas empresariales dentro del mismo ámbito, como TalentBrokers.

http://orcid.org/0000-0002-3827-1231

fumero@dit.upm.es

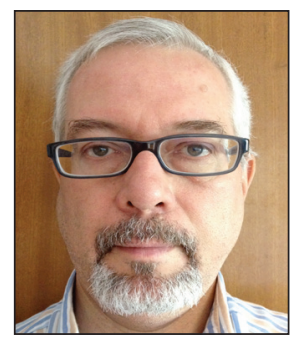

Gabriel Huecas-Fernández-Toribio es profesor del Departamento de Ingeniería Telemática (DIT) de la ETSI de Telecomunicación de la Universidad Politécnica de Madrid (UPM). Doctor ingeniero de telecomunicación por la UPM, imparte asignaturas de grado y posgrado en las áreas de transmisión de datos, técnicas de programación y desarrollo de aplicaciones móviles. Ha dirigido y participado en proyectos de investigación nacionales e internacionales, y es presidente de la Comisión de Doctorado del DIT en la UPM.

http://orcid.org/0000-0002-6725-2056

gabriel@dit.upm.es

Universidad Politécnica de Madrid ETSI de Telecomunicación Av. Complutense, 30. 28040 Madrid, España

\section{Resumen}

En el entorno tecnosocial actual la importancia de ofrecer contenidos personalizados a los usuarios de las diferentes plataformas sociales existentes, que se ajusten a sus necesidades en cada momento, es un factor clave para el éxito de las mismas. Los sistemas recomendadores juegan un papel crucial, pero en muchos casos su nivel de personalización es escaso o demasiado generalista. Se analizan dos aspectos claves para la evolución de estos sistemas: la proactividad y la contextualización. Se propone un modelo teórico de referencia para la creación de sistemas de recomendación proactivos basados en información contextual, y se comprueba su viabilidad en dos escenarios reales donde han sido implementados con éxito: el bancario y el de las redes personales de aprendizaje. Finalmente se destacan líneas de actuación futuras siguiendo las aportaciones expuestas con especial atención a su aplicación en entornos educativos.

\section{Palabras clave}

Sistemas recomendadores, Sistemas de filtraje, Proactividad, Información contextual.

Title: Proactivity and context-awareness: future of recommender systems design

\section{Abstract}

In the current techno-social environment the importance of providing personalized content for users of social platforms, continually adapting to their needs, is key to the success of the existing platforms. Recommender systems play a crucial role 
in this regard, but in many cases the level of customization provided is not enough. This article discusses two key aspects related to the development of recommender systems: proactivity and context-awareness. We propose a theoretical reference model for the creation of proactive recommender systems based on context-awareness information. In addition, we check its feasibility in two real-world scenarios where the model has been successfully implemented: banking and personal learning networks. Finally, future courses of action following the contributions proposed are highlighted, with special attention to potential applications in educational environments.

\section{Keywords}

Recommender systems, Filtering systems, Proactivity, Context-awareness.

Gallego-Vico-Daniel; Fumero-Reverón, Antonio; Huecas-Fernández-Toribio, Gabriel (2013). "Proactividad y contextualización: futuro del diseño de sistemas recomendadores". El profesional de la información, enero-febrero, v. 21, n. 1, pp.19-25.

http://dx.doi.org/10.3145/epi.2013.ene.03

\section{Introducción}

Los sistemas recomendadores (también llamados motores, marcos o plataformas de recomendación) son un tipo específico de filtrado de información que intenta recomendar artículos (películas, programas de TV, vídeos, música, libros, noticias, imágenes, páginas web, publicaciones científicas, trabajos de investigación, etc.) o elementos sociales (personas, eventos o grupos) que pueden ser de interés para un usuario (Pascual-Miguel; Chaparro-Peláez; Fumero-Reverón, 2011, p. 646).

Empezaron a ser considerados un campo de investigación con entidad propia a mediados de los años 90 del pasado siglo. Hoy gozan de cierta popularidad (Ricci; Rokach; Shapira, 2011) debido a la generalización de su uso, a lo que ha contribuido el también imparable crecimiento del comercio electrónico en cada vez más sectores y productos.

Desde el punto de vista del comerciante hay numerosas razones para integrar estos sistemas en las plataformas de comercio electrónico:

- incrementar el número y variedad de productos que se venden;

- mejorar el nivel de satisfacción de los clientes o su grado de fidelización con la marca y/o el producto;

- obtener más información acerca de sus gustos;

- mejorar la capacidad de personalización, tanto de los productos como de la propia experiencia de compra.

Los resultados de Hosanagar et al., (2008) apuntan a que la personalización que proporcionan estos sistemas ayuda a los usuarios a ampliar el rango de sus intereses, provocando efectos positivos, tanto en términos de volumen como de preferencias. Adicionalmente permite encontrar elementos comunes que faciliten la agrupación y segmentación de usuarios, y detectar tendencias en el consumo de los items que son objeto de la recomendación.

Los sistemas recomendadores se sitúan en la actualidad en un escenario caracterizado por la generalización del uso de redes sociales: el 91\% de los internautas en España tenía ya una cuenta activa en diciembre de 2011 según datos de The Cocktail Analysis (2012). En ese contexto social, los items que son objeto del proceso de recomendación son variados: por ejemplo, podemos recomendar personas, que pueden ser contactos en una comunidad de práctica o en un grupo de trabajo.

Según la misma fuente, la alta utilización de los terminales móviles inteligentes (smartphones) ha provocado que el $55 \%$ de los usuarios de internet en el móvil accedan a redes sociales a diario desde ese dispositivo. Eso hace que en el diseño de sistemas recomendadores se incorporen elementos que aprovechan la información del entorno que proporciona esa combinación de redes sociales y movilidad teniendo en cuenta además el tipo de consumo de conveniencia o compulsivo que se puede dar con mayor probabilidad en movilidad.

\section{La personalización ayuda a los usuarios a ampliar sus intereses provocando efec- tos positivos en términos de volumen y de preferencias}

En este artículo se argumenta la lógica de la introducción de estos dos elementos a partir de su evolución reciente. Se aporta un modelo teórico de referencia que será la base para eventuales implementaciones y se muestra su viabilidad empírica a través de los resultados en dos casos: en un escenario comercial, la recomendación de productos relacionados con información bancaria; y en otro social, la recomendación de contenidos educativos.

\section{Futuro de los sistemas recomendadores: proactividad y contexto}

Tradicionalmente se han agrupado en dos categorías: los basados en filtraje por contenido (content-based filtering) y los cooperativos (collaborative filtering).

Estos sistemas suelen encontrar grandes limitaciones, como por ejemplo el problema que surge al intentar generar recomendaciones para un usuario nuevo. Esta situación implica realizar el análisis de un volumen considerable de datos y lleva asociados problemas importantes de escalabilidad en los algoritmos implicados o la difícil traslación de dichos algoritmos de un ámbito concreto (por ejemplo, la recomen- 
dación de piezas audiovisuales o musicales) a otro diferente (como la recomendación de artículos textiles o productos financieros). Un problema asociado surge de la dificultad de estos sistemas para alcanzar una masa crítica de usuarios que indiquen sus preferencias, ya que sin ello se hace difícil un correcto funcionamiento del sistema recomendador. Es lo que se conoce como "arranque en frío" (cold start).

Durante los últimos años han surgido nuevos métodos que exploran la posibilidad de realizar sistemas mixtos, combinando filtraje por contenido y colaborativo (Burke, 2002). Propuestas más recientes van más allá del simple análisis de datos y añaden un análisis en tiempo real, utilizando información contextual o atendiendo a las conexiones que los usuarios establecen, mediante el estudio de grafos sociales (Woerndl; Groh, 2007).

Tal y como Pascual-Miguel, Chaparro-Peláez y Fumero-Reverón (2011) proponen, los sistemas recomendadores 2.0 deberían al menos:

1) elegir qué objeto es susceptible de ser recomendado al usuario;

2) cuándo y cómo se le debería mostrar la recomendación.

El primer punto ha sido extensamente estudiado y aplicado en muchos sistemas actuales, mientras que el segundo es una línea todavía por explorar.

\section{Tradicionalmente se han agrupado en dos categorías: los basados en filtraje por contenido (content-based filtering) y los cooperativos (collaborative filtering)}

Ricci, Rokach y Shapira (2011) señalan que la proactividad y la cada vez más rica información contextual están destinadas a cumplir un papel fundamental en la personalización de las recomendaciones de cara a definir el "cuándo" y el "cómo". Un sistema proactivo se define como aquel capaz de identificar el momento más adecuado -el "cuándo"- para generar una recomendación (Woerndl et al., 2011). Para ello se tiene en cuenta información contextual relativa al usuario (Adomavicius; Tuzhilin, 2011). Este contexto puede ser social (información relevante de conducta y contacto entre usuarios de una comunidad o red de personas), físico (factores como la ubicuidad de los dispositivos móviles y en cómo éstos afectan a la idoneidad del momento de una recomendación), de usuario (información sobre su actividad actual en tiempo real) o combinación de ellos (Gallego; Huecas; Salvachúa, 2012).

El "cómo" vendrá dado por una combinación del análisis contextual para elegir los mejores items a recomendar y de la propia experiencia de usuario frente a este tipo de sistemas proactivos. En estudios recientes (Gorgoglione; Panniello; Tuzhilin, 2011) se ha observado cómo la contextualización de la recomendación afecta positivamente al comportamiento de compra del usuario, así como a su nivel de confianza.

\section{Modelo de referencia}

Sobre la base de los trabajos realizados en Woerndl et al. (2011), se propone un modelo teórico de referencia para la creación de sistemas de recomendación proactivos basados en información contextual. En este modelo, representado en la figura 1, se separa explícitamente el "sistema de recomendación", responsable de generar las recomendaciones, y la "interfaz de usuario", que se encargará de la visualización de la recomendación generada por el primero, así como de la realimentación del mismo con la opinión del usuario.

Si nos fijamos en el bloque del "Sistema de recomendación", distinguimos tres fases:

\section{Se genera el contexto social.}

II. Se añade el contexto físico y de usuario para evaluar si la situación es adecuada para realizar la recomendación.

III. Si no se ha abortado el proceso por considerar la situación inadecuada, se realiza la evaluación de los items en función de las tendencias de consumo del usuario y de otros factores contextuales para generar la recomendación personalizada.

\section{Fase I: Generación de contexto social}

Se realiza el estudio simultáneo de todos los perfiles de usuario existentes en la plataforma o el entorno donde se ha integrado el sistema recomendador. Esta plataforma y su entorno están de hecho condicionados por la proliferación de redes sociales de propósito general, como Facebook o Twitter.

a) En primer lugar se aplican algoritmos de segmentación (clustering) sobre los perfiles de usuario existentes para generar los "clústeres sociales" que agruparán a los usuarios con los mismos intereses, generando diferentes "grafos sociales".

b) A continuación se asocian los items consumidos por cada usuario al clúster al que pertenecen. Esa asociación es la que se utilizará para definir de manera explícita el patrón o tendencia de consumo en cada agrupación o clúster.

c) Finalmente, cuando se quiera comenzar el proceso de recomendación para un usuario objetivo, si éste ya existe en la plataforma sólo se debe localizar el clúster social al que pertenece para saber su tendencia de consumo concreta. Si se trata de un usuario nuevo, se selecciona entre todos los clústeres el que guarda más similitud con él, sabiendo a partir de ese momento qué items son candidatos para dicho usuario por estar asignados al clúster social más "cercano". Este factor de similitud se calculará mediante el uso de distancias métricas adecuadas al entorno en el que el sistema ha sido desplegado y al tipo de datos que éste maneja.

\section{Fase II: Evaluación de la situación}

Para determinar el carácter proactivo del sistema, se evaluarán tres dimensiones de la información contextual del usuario:

- Social, que se ha extraído como resultado de la primera fase en forma de información de tendencias de consumo en el clúster social correspondiente al usuario. 


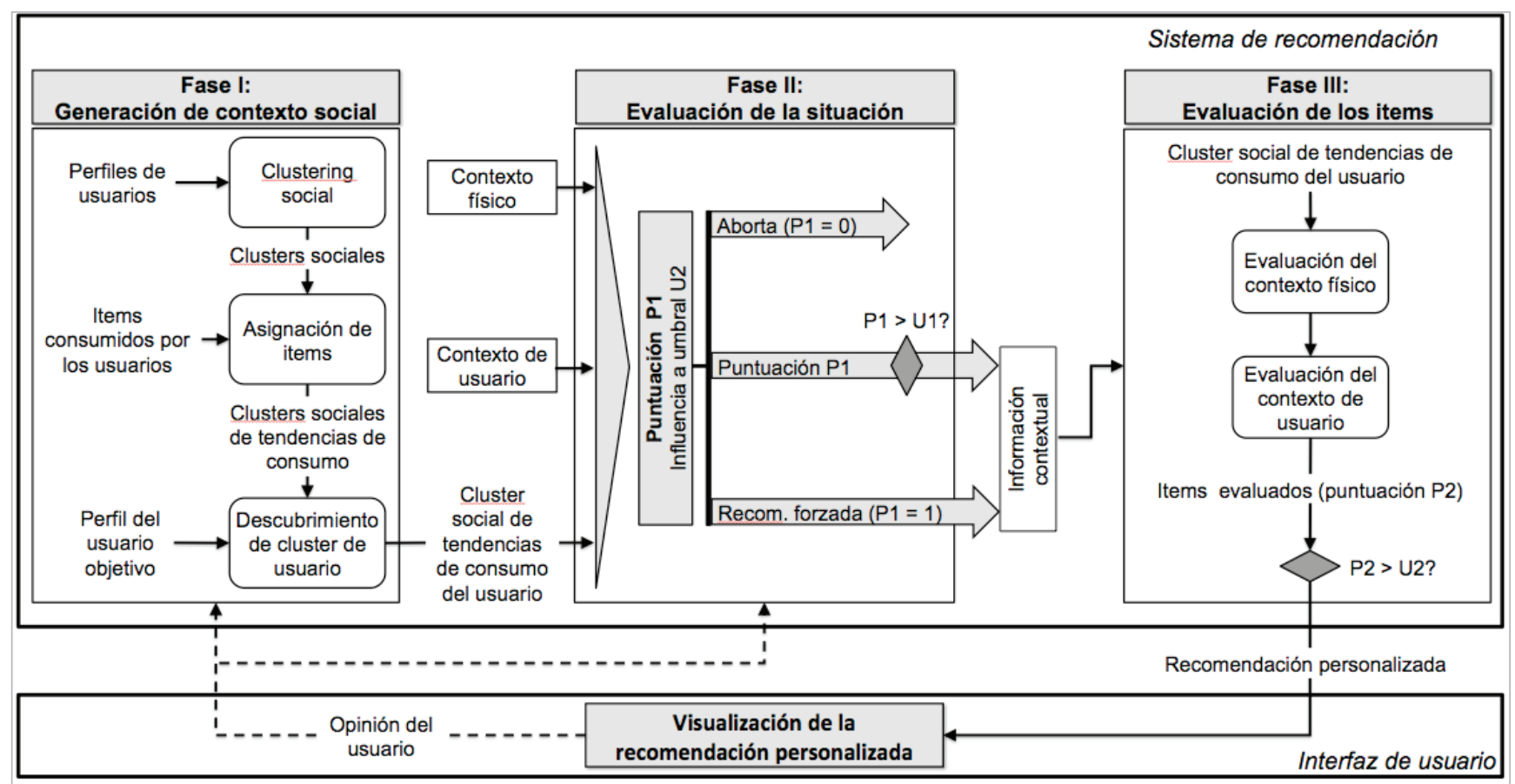

Figura 1. Modelo de recomendación proactiva basado en información contextual

- Física, incorporando elementos como su localización actual o la hora.

- De usuario, que relacionamos con la actividad concreta que el usuario está llevando a cabo en ese momento. Por ejemplo, si accede al sistema desde una plataforma móvil, o si está andando o conduciendo (mediante el estudio de parámetros de movilidad como la velocidad).

La proactividad y la información contextual están destinadas a cumplir un papel fundamental en la personalización de las recomendaciones de cara a definir el cuándo y el cómo

Una vez realizada esta evaluación, se genera una puntuación (P1 en la figura 1) que se comparará con un umbral de referencia (U1). Esto permitirá decidir si la situación actual es adecuada para generar una recomendación. Este primer umbral, responsable de determinar la proactividad del sistema, se fija inicialmente tras estudiar el escenario concreto del sistema recomendador, aunque evolucionará con el tiempo (creciendo o disminuyendo) a partir de la realimentación proporcionada por el usuario. La puntuación que determina el grado de idoneidad del momento elegido se construye estudiando los diferentes parámetros contextuales asociados a dicha situación.

\section{Fase III: Evaluación de los items}

Una vez que se ha determinado que el momento es apto para generar una recomendación, se procede a evaluar los items candidatos que vienen dados como contexto social de la fase uno. En esta fase se aplicará un proceso de evaluación donde cada tipo de contexto recibe un peso específico determinado por el escenario, tras un estudio. De esta for- ma se obtiene una puntuación para cada ítem, que será una composición de las diferentes dimensiones contextuales relativas a él.

Dicha puntuación (P2 en la figura 1) se comparará con otro umbral (U2) para decidir si ese ítem se añade a la recomendación personalizada finalmente generada. Entre U2 y P1 existirá además una dependencia inversamente proporcional tal que U2 =|1 - P1 $\mid$. Así, cuanto más adecuada sea la situación, menor será el umbral que deban superar los items evaluados en la tercera fase para ser incluidos en la recomendación personalizada.

\section{Aprendizaje del sistema: opinión del usuario}

Finalmente, en lo que se refiere a la interfaz de usuario encargada de la visualización de la recomendación, el modelo propuesto no limita en manera alguna su diseño (por ejemplo, uso de "Me gusta/No me gusta" para valorar items), pero sí destaca el importante papel que juega a la hora de permitir que el sistema "aprenda" a partir de las preferencias del usuario.

Esta realimentación al proceso de generación de la recomendación a través de opiniones del usuario afecta a las fases I y II para determinar qué items se deben recomendar, así como cuándo y cómo hacerlo. Dicho proceso de realimentación de la "opinión del usuario" en el sistema recomendador permitirá incrementar el nivel de personalización de las recomendaciones en la medida en que se use el sistema.

\section{Dos experiencias de implementación}

Llegado a este punto estamos en condiciones de particularizar el modelo de referencia en diferentes escenarios que permitirán realizar una evaluación empírica de su consistencia. A continuación sintetizaremos nuestra experiencia con dos de ellos: el bancario, con resultados consolidados; 
y el educativo, aún en desarrollo en el marco de un proyecto europeo.

\section{El banco como motor de recomendación}

Las entidades bancarias tienen acceso a un rico conjunto de datos sobre sus clientes que evita problemas importantes como el del "arranque en frío". A pesar de ello, estas entidades no suelen elaborar servicios de recomendación para sus clientes finales más allá de los que soportan los canales comerciales y la oferta de nuevos productos financieros.

Un sistema proactivo es aquel capaz de identificar el momento más adecuado -el cuándo- para generar una recomendación

Hemos tenido la oportunidad de implementar una versión simplificada de nuestro modelo teórico (figura 1) en el entorno Bankinter labs (Gallego; Huecas, 2012) dentro del marco de un proyecto de colaboración entre Bankinter y la Universidad Politécnica de Madrid. El modelo simplificado (que no incluía proactividad) se implementó en un entorno con movilidad, lo que nos permitió recabar información contextual, física y de usuarios, a través de los dispositivos móviles de los clientes. Esto dio lugar a un sistema recomendador (móvil) capaz de recomendar cualquier ítem (restaurantes, tiendas, supermercados, etc.) relacionado con una transacción bancaria de manera personalizada a cada usuario.

Los resultados devueltos tras una evaluación del sistema por parte de clientes del banco reflejaron que casi el $90 \%$ de ellos "confiaban plenamente en las recomendaciones proporcionadas", valorando además un $65 \%$ de ellos de manera positiva "la utilidad y conveniencia de implantar este tipo de sistemas recomendadores". Sólo un 5\% de los clientes mostraba una valoración desfavorable.

\section{Entornos y redes personales de aprendizaje}

La evolución de la tecnología educativa ha desembocado en el desarrollo del ámbito de lo que se ha dado en Ilamar aprendizaje tecnológicamente mediado (technology-enhanced learning, TEL). En ese campo ha cobrado cada vez más protagonismo -más allá de las particularidades de su implementación en cada nivel educativo- el concepto de "entorno personal de aprendizaje" (personal learning environment, PLE) (Grodecka; Wild; Kieslinger, 2009).

En determinados contextos, trascendiendo su origen instrumental, el concepto de PLE se ha extendido para hablar de una "red personal de aprendizaje" (personal learning network, PLN), en la que se incluyen, de forma metafórica infotecnologías, personas y contenidos (Fumero-Reverón, 2011).

El proyecto europeo "Global excursion: Extended curriculum for science infrastructure online", descrito por Holocher-Ertl, Kieslinger y Fabian (2012), tiene como objetivo principal poner en contacto a estudiantes y educadores de secunda- ria con científicos en el marco de la Unión Europea. Con ello se pretende crear un entorno para la generación y uso de contenidos educativos de mayor interés para los alumnos. Esto se logra ofreciendo acceso a través de internet a infraestructuras virtuales (e-infraestructuras) en áreas como la bio- y la nano-ingeniería, como pueden ser microscopios, experimentos de laboratorio, etc. Para ello se está desarroIlando la plataforma social Virtual science hub (ViSH).

http://www.globalexcursion-project.eu http://vishub.org

Para demostrar la viabilidad de este escenario se está implementando el modelo completo de recomendación proactiva basado en información contextual de la figura 1 como parte de la plataforma ViSH (Gallego; Barra; Aguirre; Huecas, 2012). Es de destacar el hecho de que, más allá de conseguir la eficacia requerida a la hora de generar la recomendación personalizada y contextualizada de contenidos educativos, entregando el contenido adecuado, en el momento justo para cada usuario, se está consiguiendo recomendar usuarios a partir de las agrupaciones o clústeres generados en el proceso de segmentación social, lo que está redundando en el propio proceso de crecimiento de la red social sobre la que se estructura la plataforma.

Se puede tomar como ejemplo un profesor de biología de Madrid al que se le recomienda proactivamente (a través de su móvil mientras se encuentra en horario laboral preparando sus clases), contenido educativo generado por un investigador de Cambridge que se adapta a la edad de sus alumnos; y que de ser considerado positivamente, produzca en el sistema una recomendación para establecer una colaboración más cercana entre ambos a través de una relación de "seguidor" (follower).

El cómo vendrá dado por una combinación del análisis contextual para elegir los mejores items a recomendar y de la propia experiencia de usuario frente a este tipo de sistemas proactivos

\section{Conclusiones y líneas futuras}

La evolución reciente del entorno tecnosocial en el que se integran los sistemas recomendadores justifica la necesidad de introducir nuevos elementos en su diseño. Sobre todo si tenemos en cuenta los nuevos requisitos de movilidad que han aparecido en los últimos años. Recabar y analizar información contextual del usuario y su entorno social para mejorar el proceso de recomendación es ahora un elemento clave. Además del beneficio directo que ofrece en la recomendación personalizada de diferentes productos, esa contextualización del proceso de recomendación nos permite añadir un componente proactivo.

El modelo de referencia integra ambos conceptos (proactividad y contexto) en el proceso de generación de recomendaciones. Esto ha permitido incrementar su nivel de personalización, impactando de manera positiva en la eficiencia 
de todo el sistema. Dicho modelo se ha mostrado eficaz en dos casos reales bien diferenciados (bancario y educativo), lo que se puede utilizar como un argumento en favor de su consistencia.

Los trabajos de investigación y desarrollo que se llevan a cabo en la actualidad tienen como objetivo la extensión del modelo bancario, ya consolidado, a otros casos dentro del mismo campo. El objetivo de esta línea de trabajo es soportar en el modelo diferentes perfiles de compra para un mismo usuario, evitando así "contaminaciones" en las recomendaciones debidas a compras realizadas por el usuario no destinadas a sí mismo.

La evolución reciente del entorno tecnosocial en el que se integran los sistemas recomendadores justifica la necesidad de introducir nuevos elementos en su diseño

Por otro lado, teniendo en cuenta que la componente de proactividad no se ha implementado en el escenario bancario descrito, queda como trabajo futuro evaluar de manera minuciosa el impacto de ese elemento en los clientes del banco de manera diferenciada.

El desarrollo y la ampliación del alcance del escenario educativo constituyen asimismo una línea de trabajo prometedora. Su objetivo es extender el modelo para conseguir la recomendación de objetos pedagógicos "complejos": objetos que se crean mediante la composición de objetos básicos (como imágenes, piezas de vídeo o documentos de texto).

Otra línea de trabajo abierta en este ámbito educativo busca refinar la capacidad de recomendación social de los sistemas actuales mediante la aplicación de técnicas de ciencia de redes complejas. Se trata de una línea más genérica que de hecho tendrá aplicación potencial en cualquier sistema recomendador que incluya un componente social. Es una característica que, de acuerdo con los datos que veíamos en la introducción, podemos considerar como genérica.

\section{Bibliografía}

Adomavicius, Gedimias; Tuzhilin, Alexander (2011). “Context-aware recommender systems". En: Ricci, Francesco; Rokach, Lior; Shapira, Bracha; Kantor, Paul B. Recommender systems handbook. Springer, pp. 217-253. ISBN: 9780387 858197

http://dx.doi.org/10.1007/978-0-387-85820-3_7

Burke, Robin (2002). "Hybrid recommender systems: survey and experiments". User modeling and user-adapted interaction, v. 2, n. 4, pp. 331-370.

http://dx.doi.org/10.1023/A:1021240730564

Fumero-Reverón, Antonio (2011). "IRC 2.0. Medios para la información, relación y la comunicación en la web 2.0". El profesional de la información, nov.-dic., v. 20, n. 6, pp. 605609. http://dx.doi.org/10.3145/epi.2011.nov.01

Gallego-Vico, Daniel; Huecas, Gabriel; Salvachúa, Joaquín (2012). "Generating context-aware recommendations using banking data in a mobile recommender system". En: $6^{\text {th }} \mid$ int conf on digital society, Valencia, España, pp. 73-78.

http://www.thinkmind.org/download.php?articleid=ic ds_2012_4_10_10075

Gallego-Vico, Daniel; Huecas, Gabriel (2012). “An empirical case of a context-aware mobile recommender system in a banking environment". En: $3^{\text {rd }}$ FTRA Intl conf on mobile, ubiquitous, and intelligent computing, Vancouver, Canadá, 2012, pp. 13-20.

http://dx.doi.org/10.1109/MUSIC.2012.11

Gallego-Vico, Daniel; Barra, Enrique; Aguirre, Sandra; Huecas, Gabriel (2012). "A model for generating proactive context-aware recommendations in e-learning systems". En: $42^{\text {nd }}$ ASEE/IEEE Frontiers in education conf, Seattle, EUA, pp. 1364-1369.

Gorgoglione, Michele; Panniello, Umberto; Tuzhilin, Alexander (2011). "The effect of context-aware recommendations on customer purchasing behavior and trust". En: ACM conf on recommender systems, Chicago, EUA, pp. 8592.

\section{http://dx.doi.org/10.1145/2043932.2043951}

Grodecka, Karolina; Wild, Fridolin; Kieslinger, Barbara (eds.) (2009). "How to use social software in higher education". En: iCamp Handbook, Poland. ISBN: 9788360958285 http://www.icamp.eu/wp-content/uploads/2009/01/ icamp-handbook-web.pdf

Holocher-Ertl, Teresa; Kieslinger, Barbara; Fabian, Claudia (2012). "Linking schools with science: how innovative tools can increase the effectiveness of science teaching in the classroom". En: EDEN Annual conf, Oporto, Portugal.

Hosanagar, Kartik; Fleder, Daniel M.; Lee, Dokyun; Buja, Andreas (2008). "Will the global village fracture into tribes: recommender systems and their effects on consumers". NET Institute working paper, n. 08-44, Dec 30.

http://papers.ssrn.com/sol3/papers.cfm ?abstract_ id $=1321962$

http://dx.doi.org/10.2139/ssrn.1321962

Pascual-Miguel, Félix; Chaparro-Peláez, Julián; Fumero-Reverón, Antonio (2011). "Presente y futuro de los sistemas recomendadores en la web 2.0". El profesional de la información, nov.-dic., v. 20, n. 6, pp. 645-651.

http://dx.doi.org/10.3145/epi.2011.nov.07

Ricci, Francesco; Rokach, Lior; Shapira, Bracha (2011). "Introduction to recommender systems handbook". En: Ricci, Francesco; Rokach, Lior; Shapira, Bracha; Kantor, Paul B. Recommender systems handbook. Springer, pp. 1-35. ISBN: 9780387858197

http://dx.doi.org/10.1007/978-0-387-85820-3_1

The Cocktail Analysis (2012). 4a Oleada Observatorio de Redes Sociales: Las marcas empiezan a encontrar límites, abril. http://tcanalysis.com/blog/posts/las-marcas-empiezan-aencontrar-limites-en-la-utilizacion-de-las-redes-sociales 
Woerndl, Wolfgang; Groh, Georg (2007). “Utilizing physical and social context to improve recommender systems". En: IEEE/WIC/ACM Int conf on web intelligence and intelligent agent technology workshops, Washington, EUA, pp. 123-128. http://dx.doi.org/10.1109/FWI-IATW.2007.123
Woerndl, Wolfgang; Huebner, Johannes; Bader, Roland; Gallego-Vico, Daniel (2011). "A model for proactivity in mobile, context-aware recommender systems". En: ACM conf on recommender systems, Chicago, EUA, 2011, pp. 273-276. http://dx.doi.org/10.1145/2043932.2043981

\section{http://www.elprofesionaldelainformacion.com/agenda.html}

\section{Febrero-abril}

\section{5-26 de febrero de 2013}

ASA Conference. The 3 Rs: Reach, Readership and Revenues Londres

Association of Subscription Agents and Intermediaries http://subscription-agents.org

26-27 de febrero de 2013

CONGRESO REGIONAL DE OCLC EMEA. Dynamic data: a world of possibilities

Estrasburgo

http://www.oclc.org/go/emea2013/agenda.html

5-6 de marzo de 2013

5ES JORNADES DE BIBLIOTEQUES ESCOLARS

Barcelona

COBDC, Facultat de Biblioteconomia i Documentació, Grup de Recerca Bescolar

jornades.escolars@cobdc.org

6-7 de marzo de 2013

REUNION PRESIDENCIAL IFLA 2013. Nuestro futuro digital

Mexico D.F.

IFLA Sección de América Latina y el Caribe, UNAM, AMBAC y CNB http://iibi.unam.mx/Reunion_IFLA_2013

7-9 de marzo de 2013

II JORNADAS IBEROAMERICANAS: Tecnologías digitales para la educación inclusiva y la movilidad

Úbeda, Jaén, España

José A. Ortega-Carrillo <jaorte@ugr.es>

http://fundacionugrempresa.es

13-15 de marzo de 2013

$5^{\text {TH }}$ GLOBAL CONF DIGITAL MEMORIES

Lisboa

http://www.inter-disciplinary.net/critical-issues/cyber/digital-

memories

16-17 de marzo de 2013

ICSTI'S 2013 ANNUAL MEMBERS' MEETING

Hannover

German National Library of Science and Technology-TIB; International Council for Scientific and Technical Information (Icsti) http://www.icsti.org

18 de marzo de 2013

5a JORNADA DE BIBLIOTECA PÚBLICA I COHESIÓ SOCIAL. Els joves ni... ni...

Barcelona

Biblioteques de Barcelona

http://bpcohesiosocial.wordpress.com

\section{8-19 de marzo de 2013}

CONF ON NON-TEXTUAL IN FORMATION. Strategy and innovation beyond text

Hannover

GOPORTIS

http://www.nontextualinformation2013.de
19-20 de marzo de 2013

$18^{\text {TH }}$ ANNUAL INTL CONF OF THE UKAIS. Social information systems

Oxford

UK Academy for Information Systems

http://www.ukais.org.uk/conference/conference2013

19-22 de marzo de 2013

$13^{\text {TH }}$ INTL SYMPOSIUM ON INFORMATION SCIENCE

Potsdam

University of Applied Sciences Potsdam

http://www.isi2013.de/en/isi

24-27 de marzo de 2013

$35^{\text {TH }}$ EUROPEAN CONF ON INFORMATION RETRIEVAL (ECIR 2013) Moscú

http://ecir2013.org

\section{7-30 de marzo de 2013}

WORLD CONF ON INFORMATION SYSTEMS AND TECHNOLOGIES (WORLDCIST'13)

Algarve, Portugal

AISTI (Associação Ibérica de Sistemas e Tecnologias de Informação)

http://www.aisti.eu/worldcist13

8-10 de abril de 2013

UKSG $36^{\text {TH }}$ ANNUAL CONF AND EXHIBITION

Bournemouth, Reino Unido

UKSG (antes United Kingdom Serials Group)

http://www.uksg.org/event/conference13

11-12 de abril de 2013

$5^{\text {TH }}$ EUROPEAN CONF ON INTELLECTUAL CAPITALBilbao Universidad del País Vasco

http://academic-conferences.org/ecic/ecic2013/ecic13-home. $h t m$

12-14 de abril de 2013

GALLERIES, LIBRARIES, ARCHIVES, MUSEUMS \& WIKIMEDIA (GLAM-WIKI) 2013

Londres

Wikimedia UK

http://uk.wikimedia.org/wiki/GLAM-WIKI_2013

21-27 de abril de 2013

$69^{\text {TH }}$ FIAF CONGRESS

Barcelona

Intl. Federation of Film Archives, Filmoteca de Catalunya http://www.fiafcongress.org/2013

22-24 de abril de 2013

$6^{\text {TH }}$ INTL CONF ON THE INCLUSIVE MUSEUM. Museums and active citizenship

Copenhague

National Art Gallery of Denmark

http://onmuseums.com 